\title{
Comparison the effects of oxytocin and methylergonovine in elective caesarean section under spinal anesthesia
}

\author{
Abdorasoul Anvaripour • Houshang Shahryari • \\ Shahnaz Ahmadi · Soghra Ghasemi · \\ Kamran Mirzaei
}

Received: 23 July 2012/Accepted: 3 December 2012/Published online: 19 December 2012

(c) Springer-Verlag Berlin Heidelberg 2012

\begin{abstract}
Purpose In order to prevent postpartum hemorrhage in caesarean section under spinal anesthesia, patients routinely receive oxytocin. In this study we compared the efficacy of Methylergonovine and Oxytocin on hemodynamic stability and bleeding amount in caesarean section. Materials and methods In this randomised controlled trial study, 80 patients candidate for elective caesarean section under spinal anesthesia divided to two groups: 40 patients in control group received oxytocin and 40 ones in case group received methylergonovine.

Results There was no differences between groups in Mean age, baseline hemodynamic values, after spinal anesthesia and recovery (except diastolic blood pressure $\min 20$ ), time of uterine atony, dizziness; nausea and vomiting. After drug administration (oxytocin and methylergonovine), systolic blood pressure in minutes 1, 10, 15 and diastolic blood pressure in minutes 1, 3, 20 increased in case group statistically more than control group. In control group, heart rate in minutes 1,5 increased significantly
\end{abstract}

A. Anvaripour $\cdot$ H. Shahryari

Anesthesiology Department, University

of Medical Sciences, Bushehr, Iran

\section{A. Anvaripour}

The Persian Gulf Tropical Medicine Research Center, Bushehr University of Medical Sciences, Bushehr, Iran

S. Ahmadi $(\bowtie) \cdot$ S. Ghasemi

Gynecology Department, University

of Medical Sciences, Bushehr, Iran

e-mail: Ahmadishahnaz2005@yahoo.com

K. Mirzaei

Department of Community Medicine,

University of Medical Sciences, Bushehr, Iran more than the other group. Mean arterial blood pressure in minutes $1,3,5,10,15$ reduced significantly more than in control group. Need to vasoconstrictor drug statistically was less in case group $(p<0.0001)$.

Conclusion Methylergonovine induced significantly more hemodynamic stability. Adverse effects were similar between two groups. We recommend the use of methylergonovine in patients with caesarean section under spinal anesthesia because of its hemodynamic stability and low need to vasoconstrictor drugs.

Keywords Methylergonovine - Oxytocin · Hemodynamic . Spinal anesthesia $\cdot$ Elective cesarean

\section{Introduction}

Today, Caesarean section is the most common surgery in the United States [1], and both regional and general anesthesia are commonly used in these procedures [2].

The American College of Obstetrics and Gynecology has concluded that regional anesthesia is the preferred choice to control labor pain and should be used if no contraindication is present [3]. Hypotension (systolic blood pressure less than $90 \mathrm{mmHg}$ ) is seen in nearly $33 \%$ of patients under spinal anesthesia, and it is the most common complication of spinal anesthesia for delivery [4].

Postpartum hemorrhage (PPH) is one of the four main causes of maternal mortality due to delivery after 20 weeks of gestational age (in addition to thromboembolism, hypertensive disorders, and infection) [1]; it is probably the most common one [1]. Uterine atony-inability of the uterus to contract properly after delivery-is the most prevalent cause of PPH [3]. The incidence of uterine atony is on the increase in both vaginal delivery and cesarean 
section [5]. Oxytocin is still the first-line uterotonic drug for prophylaxis and treatment of uterine atony [5]. A study by Jonsson et al. [6] has shown that bolus dosage of oxytocin induces transient hypotension and reflex tachycardia and increases cardiac output in healthy women undergoing elective caesarean section under spinal anesthesia; these effects are dose-dependent. Moreover, systemic hemodynamic changes or coronary vasospasm lead to myocardial ischemia and electrocardiogram (ECG) changes [6]. The effects of oxytocin are limited by the numbers and situation of oxytocin receptors. It is worth mentioning that increasing the dosage does not necessarily improve uterine contractions if numbers and quality of the receptors are inadequate [7]. Therefore, the other uterotonic agents, with different mechanisms, could be used instead [7]. Methylergonovine continues to be the second-line uterotonic agent in the prophylaxis and treatment of PPH [5]. Methylergonovine is a synthetic alkaloid that causes strong contractions of the myometrium; however, parenteral administration of this alkaloid, especially via the intravenous route, sometimes causes transient but severe elevated blood pressure [3]. Some studies have shown that oxytocin plus ergometrine is more effective than oxytocin alone in the prevention of PPH [8-10].

The objective of this study was to compare the efficacy of methylergonovine vs. oxytocin on hemodynamic stability and bleeding amount in cesarean section under spinal anesthesia. The study also considered side effects and time of atony.

\section{Materials and methods}

A prospective, randomized, single-blind clinical trial was conducted on 80 women candidates for elective cesarean section, under spinal anesthesia, who were admitted to Bentolhoda Obstetrics and Gynecology Hospital in Bushehr, Iran. Written informed consent was obtained from all patients after full explanation of the procedure, and the study was approved by the institutional Ethical Review Board.

The inclusion criterion was first pregnancy after 18 years of age.

Exclusion criteria were positive history of cardiovascular disease, positive history of preeclampsia, allergy to ergot alkaloids, uterine atony lasting more than five minutes, general anesthesia, chronic hypertension, emergent cesarean section, and factors increasing the risk of atony, including prolonged labor, polyhydramnios, multiparity, and large baby.

Patients were randomized into two groups using computer-generated randomizing schedules. While the patients were entering the operation room, an intravenous line was fixed, and $500 \mathrm{~mL}$ isotonic serum $(0.9 \%$ saline $)$ was administrated to each one. Baseline hemodynamic values-systolic blood pressure, diastolic blood pressure, heart rate, and mean arterial blood pressure measured by non-invasive blood pressure (NIBP) apparatus-were recorded, using a checklist.

A single dose of intravenous metoclopramide $(10 \mathrm{mg})$ was administrated to all the patients three minutes before spinal anesthesia.

Spinal anesthesia was performed on all the patients while in a sitting position, using $12 \mathrm{mg}$ hyperbaric intraarachnoidal procainamide $0.5 \%$ (Milan, Italy) and 25 microgram fentanyl (Aburaihan Pharmaceutical Co., Tehran, Iran) via 25-26 gauge Barter spinal needle (Dr. Japan Co., Japan) in the subarachnoid space between the L3-L4 or L4-L5 vertebrae.

In the 1st, 3rd, and 5th min after spinal anesthesia, the patients were monitored while in a supine position by NIBP apparatus, and systolic and diastolic blood pressure, heart rate, and mean arterial pressure were recorded.

If the systolic blood pressure decreased less than $90 \mathrm{mmHg}, 10 \mathrm{mg}$ intravenous ephedrine was injected as a vasoconstrictor agent.

When the level of anesthesia reached T6, the Cesarean section was initiated.

Thirty minutes after delivery, $0.2 \mathrm{mg}$ methylergonovine (Minoo Co, Tehran, Iran) in $1 \mathrm{~L}$ isotonic serum in the case group, and 30 units oxytocin (Caspiantamin Pharmaceutical Co, Tehran, Iran) in $1 \mathrm{~L}$ isotonic serum in the control group, were infused by an anesthesiologist. After drug administration, systolic and diastolic blood pressure, heart rate, and mean arterial pressure were recorded on the checklist at 1, 3, 5, 10, 15, and $20 \mathrm{~min}$, and every $5 \mathrm{~min}$ until the end of the operation.

After surgery, all patients were transferred to a recovery room. Primary outcome included amount of bleeding, which was estimated by the amount in the field of surgery and the amount of aspirated blood after suctioning amniotic fluids into the bottle of a suction apparatus separately. Secondary outcomes were systolic and diastolic blood pressure, heart rate, and mean arterial pressure, which were recorded on the checklist while entering the recovery room and at 5, 10, 15, 20, and $30 \mathrm{~min}$. Incidence of dizziness, nausea, vomiting, and the need for ephedrine as a vasoconstrictor were recorded during the surgery and in the recovery room.

In order to detect differences with a $5 \%$ level of significance, $90 \%$ power and a standard difference $1.0 \mathrm{sam}$ ple size calculation estimated 80 patients by using the Altman curve (http://ccforum.com/content/6/4/335).

Statistical analysis

Statistical analysis was performed with SPSS software (version 16). Due to the non-parametric distribution of 
data, statistical significance for the differences was tested by Chi-square for comparing frequencies, and MannWhitney and the Kruskal-Wallis test for comparing mean of variables, when appropriate. A $p$ value less than 0.05 was considered statistically significant.

\section{Results}

Eighty patients met the inclusion criteria and were randomly assigned to two groups. The 40 patients in the case group received methylergonovine, and the 40 patients in the control group received oxytocin.

No differences were found between the groups in terms of mean age or baseline hemodynamic values, including systolic and diastolic blood pressure, heart rate, and mean arterial pressure (Table 1).

In addition, no differences were found between the groups in terms of systolic and diastolic blood pressure, heart rate, and mean arterial pressure after spinal anesthesia at 1,3 , and $5 \min (p>0.05)$.

After drug injection (oxytocin or methylergonovine), statistically significant differences were found between the two groups in terms of systolic and diastolic blood pressure, heart rate, and mean arterial pressure. Heart rate in the oxytocin group was statistically higher at 1 and $5 \mathrm{~min}$. Mean arterial pressure in the oxytocin group was statistically lower in minutes $1,3,5,10$, and 15 . Systolic blood pressure at minutes $1,10,15$, and 20, and diastolic blood pressure at minutes 1,3 , and 20 were statistically higher in the methylergonovine group (Table 2).

No statistical significance was discovered between the two groups in terms of systolic blood pressure, heart rate, or mean arterial pressure during recovery $(p>0.05)$; only diastolic blood pressure was statistically higher in the methylergonovine group in minute 20 in the recovery room $(p=0.03)$.

Table 1 Comparison of mean age and baseline hemodynamic values between the two groups $(n=40)$

\begin{tabular}{llll}
\hline Parameter & $\begin{array}{l}\text { Oxytocin } \\
\text { group } \\
\text { Mean (SD) }\end{array}$ & $\begin{array}{l}\text { Methylergonovin } \\
\text { group } \\
\text { Mean (SD) }\end{array}$ & $p$ value \\
\hline $\begin{array}{l}\text { Mean age } \\
\text { (years) }\end{array}$ & $27.45 \pm 4.81$ & $28.08 \pm 5.13$ & 0.06 \\
SBP (mmHg) & $130.83(18.21)$ & $128.60(12.66)$ & 0.54 \\
DBP (mmHg) & $83.51(10.46)$ & $83.76(14.33)$ & 0.93 \\
HR (beat/min) & $101.92(19.21)$ & $101.92(19.21)$ & 0.35 \\
MAP (mmHg) & $94.77(15.30)$ & $95.68(14.94)$ & 0.80 \\
\hline
\end{tabular}

$\overline{S B P}$ systolic blood pressure, $D B P$ diastolic blood pressure, $H R$ heart rate, $M A P$ mean arterial pressure

* Statistical test: Mann-Whitney
As shown in Table 3, women in the methylergonovine group had a significantly lower need for ephedrine as a vasoconstrictor drug $(p<0.0001)$.

No differences were observed between the groups in terms of bleeding amount, nausea and vomiting, dizziness, or time of atony during the operation and in recovery (Table 3).

\section{Discussion}

The cardiovascular effects of oxytocin in animal models and women undergoing Caesarean section include tachycardia, hypotension, and decreased cardiac output. These factors can be sufficient to cause significant compromise in high-risk patients.

This study demonstrated that the need for ephedrine as a vasoconstrictor drug in the methylergonovine group was

Table 2 Comparison mean of hemodynamic values in minutes after oxytocin and methylergonovine injection between the two groups $(n=40)$

\begin{tabular}{|c|c|c|c|}
\hline Parameter & $\begin{array}{l}\text { Oxytocin group } \\
\text { Mean (SD) }\end{array}$ & $\begin{array}{l}\text { Methylergonovin } \\
\text { Mean (SD) }\end{array}$ & $p$ value ${ }^{*}$ \\
\hline HR1 (b/m) & $103.60(15.5)$ & $95.38(15.04)$ & 0.01 \\
\hline HR3 (b/m) & $100.85(15.12)$ & $95.90(18.69)$ & 0.19 \\
\hline HR5 (b/m) & $102.60(15.64)$ & $94.76(17.65)$ & 0.04 \\
\hline HR10 (b/m) & $102.18(13.80)$ & 97.07 (13.93) & 0.10 \\
\hline HR15 (b/m) & $99.00(12.51)$ & $97.13(14.60)$ & 0.53 \\
\hline HR20 (b/m) & $95.24(13.12)$ & 93.49 (14.09) & 0.57 \\
\hline SBP1 (mmHg) & 112.05 (12.98) & $120.85(20.56)$ & 0.03 \\
\hline SBP3 (mmHg) & $110.90(14.47)$ & 114.17 (13.76) & 0.30 \\
\hline SBP5 (mmHg) & $108.93(12.58)$ & 110.90 (11.67) & 0.46 \\
\hline SBP10 (mmHg) & $106.85(12.83)$ & 115.75 (16.27) & 0.00 \\
\hline SBP15 (mmHg) & $108.43(12.48)$ & $114.75(10.87)$ & 0.01 \\
\hline SBP20 (mmHg) & 111.79 (12.69) & $118.15(12.40)$ & 0.02 \\
\hline DBP1 (mmHg) & $63.12(12.19)$ & $69.72(12.39)$ & 0.01 \\
\hline DBP3 (mmHg) & $60.30(12.14)$ & $60.87(10.31)$ & 0.30 \\
\hline DBP5 (mmHg) & $58.35(11.60)$ & $62.10(10.80)$ & 0.13 \\
\hline DBP10 (mmHg) & $55.59(12.47)$ & $75.35(7.48)$ & 0.12 \\
\hline DBP15 (mmHg) & $58.35(12.36)$ & $62.15(8.03)$ & 0.10 \\
\hline DBP20 (mmHg) & $61.92(11.69)$ & $68.85(12.14)$ & 0.01 \\
\hline MAP1 (mmHg) & $75.72(12.08)$ & $85.84(12.13)$ & 0.00 \\
\hline MAP3 (mmHg) & $75.26(13.11)$ & $81.59(9.87)$ & 0.01 \\
\hline MAP5 (mmHg) & 73.95 (12.22) & 78.79 (10.02) & 0.05 \\
\hline MAP10 (mmHg) & 70.95 (11.44) & $79.92(13.22)$ & 0.00 \\
\hline MAP15 (mmHg) & 72.33 (10.75) & $79.69(12.65)$ & 0.00 \\
\hline MAP20 (mmHg) & 93.77 (10.29) & 84.76 (12.29) & 0.58 \\
\hline
\end{tabular}

$\mathrm{b} / \mathrm{m}$ beat/minute, $S B P$ systolic blood pressure, $D B P$ diastolic blood pressure, $H R$ heart rate, $M A P$ mean arterial pressure

* Statistical test: Mann-Whitney 
Table 3 comparison of complications between the two groups

\begin{tabular}{lllc}
\hline Parameter & Oxytocin group & $\begin{array}{l}\text { Methylergonovin } \\
\text { group } \\
n=40\end{array}$ & $p$ value \\
\hline $\begin{array}{l}\text { Hypotension \& } \\
\begin{array}{l}\text { Ephedrine } \\
\text { injection }\end{array}\end{array}$ & $57.5 \%$ & $20 \%$ & $<0.0001^{*}$ \\
$\begin{array}{l}\text { Bleeding while } \\
\text { operation (mL) }\end{array}$ & $452.25 \pm 120.18$ & $433.25 \pm 108.44$ & $0.46^{* *}$ \\
$\begin{array}{l}\text { N/V while } \\
\text { operation }\end{array}$ & $32.5 \%$ & $22.5 \%$ & 0.32 \\
$\begin{array}{l}\text { N/V in recovery } \\
\text { Dizziness }\end{array}$ & $5 \%$ & $7.5 \%$ & 0.65 \\
$\begin{array}{l}\text { Time of atony } \\
\text { (min) }\end{array}$ & $4.13 \pm 3.11$ & $3.87 \pm 2.41$ & 0.81 \\
\hline
\end{tabular}

N/V Nausea and vomiting, $\min$ minute

* Chi-square

** Mann-Whitney

significantly less than in the oxytocin group. No differences were found between the two groups in terms of nausea, vomiting, and dizziness.

Clinical outcomes in a study by Fujimoto et al. [10] revealed that intramuscular administration of oxytocin, compared with methylergometrine, was associated with a significant reduction in postpartum blood loss and in frequency of blood loss of more than $500 \mathrm{~mL}$.

Another study, by Saito et al. [11], demonstrated that the routine use of intramuscular oxytocin was more effective than the use of intramuscular ergometrine for prevention of postpartum hemorrhage in the third stage of labor. There were no differences between the groups in terms of mean duration of the third stage, mean level of hemoglobin on the second postpartum day, frequency of postpartum hemorrhage $(\geq 1,000 \mathrm{~mL})$, or manual removal of placenta. Fewer side effects were found, with no significant differences between the groups [11].

The results of our study differed from the Fujimoto and Saito investigations. In our study, there were no differences between the groups in terms of bleeding during the operation. This difference could be due to a difference in the type of delivery and dosage of the drug administered. In addition, in our study, methylergonovine and oxytocin were infused intravenously, which was the novelty of our study.

In a study by Kikutani et al. [12], the amount of blood loss in the oxytocin groups were significantly lower than in the methylergonovine group during Caesarean section under spinal anesthesia. In our study, there was no significant difference in amount of blood loss between the groups.

However, in a study by Howard et al. [13], which was conducted to assess the effects of oxytocin, methylergonovine maleate, and a placebo, upon the fourth stage of labor, methylergonovine maleate was the agent most effective in preventing postpartum hemorrhage.

In a study by Svanström et al. [14], which was conducted to compare oxytocin and methylergometrine during Caesarean section under spinal anesthesia, oxytocin administered as an intravenous bolus of $10 \mathrm{IU}$ induced chest pain, transient profound tachycardia, hypotension, and concomitant signs of myocardial ischemia according to marked ECG changes, while methylergometrine produced mild hypertension and no significant ECG changes. This study confirms the results of our study, in which tachycardia and decreased mean arterial pressure after oxytocin administration was observed.

In a study by Singh et al. [15], which was conducted to compare sublingual misoprostol, intravenous oxytocin, and intravenous methylergometrine in active management of the third stage of labor, increased blood pressure was observed significantly in the methylergometrine group in comparison with the other groups.

In our study, after infusion of oxytocin and methylergonovine during surgery, systolic blood pressure in the methylergonovine group in minutes $1,10,15$, and 20 , and diastolic blood pressure in minutes 1, 3, and 20, were statistically higher than in the oxytocin group. These hemodynamic changes in the methylergonovine group demonstrate the relative stability of the hemodynamic parameters in this group at those times.

There were two limitations to our study. The first limitation was patients' refusal for spinal anesthesia, as it is an absolute contraindication for spinal anesthesia, and the second was the limitation to selected primipara patients who were candidates for elective Caesarean section.

This pilot study was designed to assess the effects of methylergonovine in Caesarean section. Another, larger clinical trial is required to determine the influence of methylergonovine in Caesarean section.

In conclusion, our findings demonstrate that intravenous infusion of methylergonovine can be applied instead of intravenous oxytocin in elective Caesarean section under spinal anesthesia, because it induces more hemodynamic stability, prevents hypotension, and decreases the need for vasoconstrictor drugs due to hypotension. In addition, the side effects are not statistically different from those associated with oxytocin.

\section{Conflict of interest None.}




\section{References}

1. Ronald S, Gibbs DN et al (2008) Complications of delivery. Danforth's Obstetrics and Gynecology, 10th edn. Lippincott Williams \& Wilkins, Philadelphia, pp 290-291

2. Afolabi BB, Lesi FEA, Merah NA (2007) Regional versus general anaesthesia for caesarean section. Cochrane Database Syst Rev. doi: 10.1002/14651858

3. Moertl MG, Friedrich S, Kraschl J et al (2011) Hemodynamic effects of carbetocin and oxytocin given as intravenous bolus on women undergoing caesarean delivery: a randomized trial. BJOG 118(11):1349-1356

4. Miller RD, Stoeltieng RK (2007) Basics of Anesthesia, 5th edn. Churchill Livingstone, Philadelphia, pp 280-281

5. Manrique Munoz S, Munar Bauza F, Frances Gonzalez S et al (2012) Update on the use of uterotonic agents. Rev Esp Anestesiol Reanim 59:91-97

6. Jonsson M, Hanson U, Liddle C et al (2010) ST depression at cesarean section and the relation to oxytocin dose: a randomized Controlled trial. BJOG 117:76-83

7. Balki M, Dhumne S, Kasodekar S et al (2008) Ergot and oxytocin during cesarean delivery following failure to progress in labour. BJOG 115:579-840

8. Kumru S, Gurates B, Parmaksiz C (2005) Investigation of the usefulness of methylergonovin application in cesarean section cases. J Turkish German Gynecol Assoc. 6:42-45
9. Mitchell GG, Elboume DR (1993) The salford third stage trial. Oxytocin plus ergometrine versus oxytocin alone in the active management of the third stage of labor. Online J Curr Clin Trials (doc no 83: 2305 words; 32 paragraphs)

10. Fujimoto M, Takeuchi K, Sugimoto M et al (2006) Prevention of postpartum hemorrhage by uterotonic agents: comparison of oxytocin and methylergometrine in the management of the third stage of labor. Acta Obstet Gynecol Scand 85:1310-1314

11. Saito K, Haruki A, Ishikawa H et al (2007) Prospective study of intramuscular ergometrin compared with intramuscular oxytocin for prevention of postpartum hemorrhage. J Obstet Gynaecol Res $33: 254-258$

12. Kikutani T, Kikutani M, Oshima M et al (2006) Effects of methylergonovine and oxytocin on blood loss and uterine contraction during cesarean section. Masui 55(5):590-594

13. Howard WF, McFadden PR, Keettel W (1964) Oxytocic drugs in fourth stage of labor. JAMA 189:411-413

14. Svanström MC, Biber B, Hanes M et al (2008) Signs of myocardial ischaemia after injection of oxytocin: a randomized double-blind comparison of oxytocin and methylergometrine during caesarean section. Br J Anaesth 100:683-689

15. Singh G, Radhakrishnan G, Guleria K (2009) Comparison of sublingual misoprostol, intravenous oxytocin, and intravenous methylergometrine in active management of the third stage of labor. Int J Gynaecol Obstet 107:130-134 